\title{
Direct Measurement of Intramolecular Electron Transfer in a Series of Artificial Photosynthesis Processes
}

\author{
Ken Onda ${ }^{1,}{ }^{*}$, Kei Ohkubo ${ }^{2}$, Yasuomi Yamazaki ${ }^{2}$, Kazuhide Koike $^{3}$, Sei'ichi Tanaka ${ }^{2}$, and \\ Osamu Ishitani $^{2}$ \\ ${ }^{1}$ Department of Chemistry, Kyushu University, Nishi-ku, Fukuoka 819-0395, Japan \\ ${ }^{2}$ Department of Chemistry, Tokyo Institute of Technology, Meguro-ku, Tokyo 152-8550, Japan \\ ${ }^{3}$ National Institute of Advanced Industrial Science and Technology, Tsukuba, 305-8569, Japan
}

\begin{abstract}
We have directly determined the intramolecular electron transfer rate and revealed its mechanism in supramolecular complexes during $\mathrm{CO}_{2}$ photoreduction by time-resolved infrared spectroscopic measurements over a wide temporal range.
\end{abstract}

\section{Introduction}

Artificial photosynthesis is a chemical process for creating high-energy molecules from $\mathrm{CO}_{2}$ as a carbon source and $\mathrm{H}_{2} \mathrm{O}$ as an electron source using sunlight. However, no examples of practical artificial photosynthesis have been realized yet, though many attempts have been made as part of the effort to address the global energy problem. One difficulty is that a photosynthesis system is composed of multi-step chemical processes involving many chemical species. The best method to understand these processes is the direct measurement of intermediate species using ultrafast spectroscopy. However, few studies have been made compared to biological photosynthesis. We have studied artificial photosynthesis using time-resolved infrared vibrational (TR-IR) spectroscopy in a wide temporal range from femtoseconds to microseconds, which makes it possible to identify chemical species step by step even in mixed solutions [1-7].

Electron transfer from a photosensitizer to a reaction center is one of the most important processes in artificial photosynthesis. However, it has never been directly observed and its mechanism is unknown because the process occurs during the course of multi-step chemical reactions. In the $\mathrm{CO}_{2}$ reduction photocatalytic reaction, electron transfer occurs after electron injection from a reductant triggered by photoexcitation of the photosensitizer. Further processes are involved before $\mathrm{CO}_{2}$ is reduced to $\mathrm{CO}$, and the temporal range of the overall processes is from femtoseconds to microseconds [1,8]. We studied this series of chemical reactions using TR-IR spectroscopy and evaluated the electron transfer rates in various supramolecular complexes having different bridging ligands. From these rate constants, we determined the mechanism for intramolecular electron transfer in the supramolecular complexes.

\footnotetext{
* Corresponding author: konda@chem.kyushu-univ.jp
} 


\section{Experimental}

TR-IR spectra over a wide temporal range were measured by a pump-probe method using a femtosecond Ti:sapphire amplifier and a sub-nanosecond $\mathrm{Nd}: \mathrm{YVO}_{4}$ laser. A mid-infrared probe pulse (pulse duration $=120 \mathrm{fs}$, tuneable range $=1000-4000 \mathrm{~cm}^{-1}$ ) was generated by optical parametric amplification (OPA) and difference-frequency generation from part of the output of the amplifier. The probe pulse passed through an infrared flow cell and was detected by a mid-infrared detector array equipped with a polychromator. The 532-nm pump pulse was generated by OPA and sum-frequency generation from the other part of the output of the amplifier. The temporal delay between the pump and probe pulses was from $100 \mathrm{fs}$ to $1.2 \mathrm{~ns}$ and was created by an optical delay line. For measurements more than $1 \mathrm{~ns}$, another 532-nm pump pulse was generated by second harmonic generation of the output of the sub-nanosecond laser (wavelength $=1064 \mathrm{~nm}$, pulse duration $=600 \mathrm{ps}$ ), which was electronically synchronized with the amplifier with a timing jitter of $<200$ ps.

\section{Results and Discussion}

The supramolecular complexes under study were Ru-Re complexes, consisting of a Ru unit as a photosensitizer, a Re unit as a reaction center, and a bridging ligand. Figure 1 shows the reaction scheme for the early stage of $\mathrm{CO}_{2}$ photoreduction for this type of supramolecular complex. Briefly, electron transfer occurs from the one-electron reduced $\mathrm{Ru}$ unit $\left(\mathrm{Ru}^{-}\right)$to the Re unit, followed by electron injection from the strong reductant $(\mathrm{BIH})$ to the photo-excited $\mathrm{Ru}$ unit $\left({ }^{3} \mathrm{Ru}^{*}\right)$. Typical concentrations of $\mathrm{Ru}-\mathrm{Re}$ and $\mathrm{BIH}$ were $0.2 \mathrm{mM}$ and $0.3 \mathrm{M}$, respectively, in a mixed solvent DMF-TEOA $(5: 1 \mathrm{v} / \mathrm{v})$.

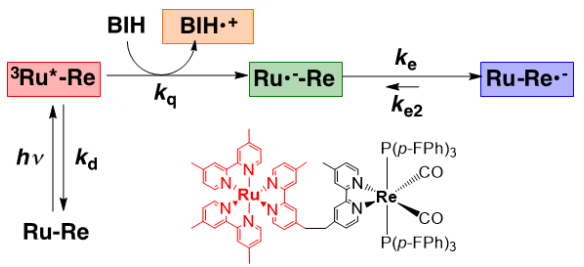

Fig. 1. Reaction scheme for the early stage of $\mathrm{CO}_{2}$ photoreduction using $\mathrm{Ru}-\mathrm{Re}$ supramolecular complex. $k_{\mathrm{d}}, k_{\mathrm{q}}, k_{\mathrm{e}}$, and $k_{\mathrm{e} 2}$ are the rate constants for each process.

Figure 2a shows examples of TR-IR spectra for this reaction in a complex with a $\mathrm{C}_{2} \mathrm{H}_{4}$ bridging ligand (C2). The transient absorption bands at 1836 and $1907 \mathrm{~cm}^{-1}$ are assigned to a complex with a reduced $\mathrm{Re}$ unit $\left(\mathrm{Ru}_{-} \mathrm{Re}^{-}{ }^{-}\right)$[9]. Since the intensities of these bands correspond to the amount of this species, we plot one of them as a function of delay time as black dots in Fig. 2b. To reproduce this temporal profile, we set up the rate equations according to the scheme in Fig. 1 [1]. The rate constants $k_{\mathrm{d}}$ and $k_{\mathrm{q}}$ were determined to be $1.34 \times 10^{6} \mathrm{~s}^{-1}$ and $1.2 \times 10^{9} \mathrm{M}^{-1} \mathrm{~s}^{-1}$ based on the luminescence lifetimes of the Ru unit and the dependence of the quenching rate on the reductant concentration (Stern-Volmer plot), respectively. In addition, the ratio $k_{\mathrm{e} 2} / k_{\mathrm{e}}$ was determined to be 9 from the UV/Vis spectra for a photo-steady state. Thus, only one parameter remains undetermined, the electron transfer rate from the $\mathrm{Ru}$ unit to the Re unit, $k_{\mathrm{e}}$. We carried out a least-squares fit by solving the rate equations using these rate constants and determined the electron transfer rate to be $k_{\mathrm{e}}=1.4 \times 10^{9} \mathrm{~s}^{-1}$. The obtained concentrations of $\mathrm{Ru}-\mathrm{Re}^{--}$, the complex with a reduced $\mathrm{Ru}$ unit $\left(\mathrm{Ru} \cdot{ }^{-}-\mathrm{Re}\right)$, and the complex with a photoexcited $\mathrm{Ru}$ unit $\left({ }^{3} \mathrm{Ru}{ }^{*}-\mathrm{Re}\right)$ are indicated by blue, green, and red lines in Fig. 2b, respectively. We repeated these measurements and calculations for another four supramolecular complexes having different bridging ligands, 
$\mathrm{RC} 2, \mathrm{C} 4, \mathrm{COC}$, and $\mathrm{C} 6$, and plotted the logarithm of the rate constants as a function of the bond length between the Ru and Re units along the bridging ligand $(r)$ in Fig. 2c. We found a linear correlation between these values except for the RC2 bridging ligand, which is exceptional because it is a double bridging ligand.

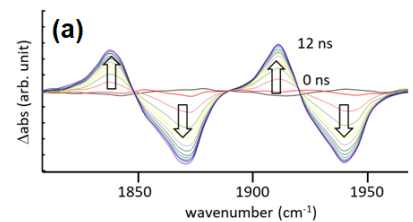

(c)
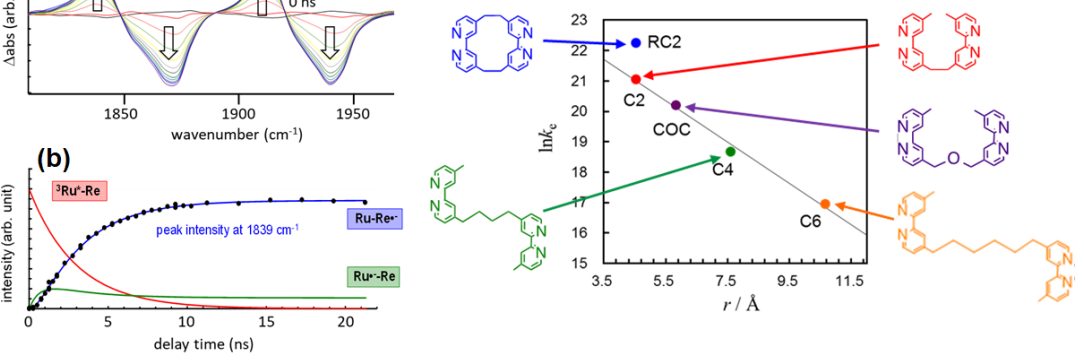

Fig. 2. (a) Selected TR-IR spectra measured while the reaction in Fig. 1 occurs. (b) Temporal profiles of peak intensity at $1839 \mathrm{~cm}^{-1}$ and concentrations simulated by solving the rate equations. (c) Logarithmic plot of $k_{\mathrm{e}}$ vs. bond length for five different bridging ligands. The insets show the structures of the bridge ligands.

According to semi-classical theory [10], the electron transfer rate, $k_{\mathrm{e}}$, is expressed by the equation: $k_{\mathrm{e}} \propto \exp \left(-\beta\left(r-r_{0}\right)\right)$, where $\beta$ is a coefficient factor, and $r$ and $r_{0}$ are the distance and the close contact distance between the donor and the acceptor, respectively. It is known that the coefficient $\beta$ strongly depends on the mechanism of the electron transfer, for example, $\beta \sim 3.4 \AA^{-1}$ for through-space [11], $\beta \sim 0.8-1.0 \AA^{-1}$ for through-alkyl-bond [10,12]. Our plot of $k_{\mathrm{e}}$ vs. $r$ in Fig. 2c shows good exponential correlation, except for RC2. From this the coefficient factor was determined to be $\beta \sim 0.67 \AA^{-1}$. Because this value is close to 0.8-1.0 $\AA^{-1}$, we concluded that the electron transfer occurs by a through-alkyl-bond mechanism. Intriguingly, this result indicates that the flexibility of the bridging ligand does not affect the electron transfer, although it is possible that the two units directly collide with each other when they are connected by a long alkyl chain such as C6.

\section{References}

1. K. Koike, D.C. Grills, Y. Tamaki, et al. Chem. Sci. 9, 2961 (2018)

2. T. Mukuta, P.V. Simpson, J.G. Vaughan, et al. Inorg. Chem. 56, 3404 (2017)

3. S. Tanaka, Y. Matsubara, T. Asatani, et al. Chem. Phys. Lett. 662, 120 (2016)

4. T. Mukuta, S. Tanaka, A. Inagaki, et al. ChemistrySelect, 1, 2802 (2016)

5. S. Tanaka, K. Takahashi, et al. J. Photochem. Photobio. A: Chem. 313, 87 (2015).

6. T. Mukuta, N. Fukazawa, K. Murata, et al. Inorg. Chem. 53, 2481 (2014)

7. S. Sato, Y. Matsubara, K. Koike, M. et al. Chem. Eur. J. 18, 15722 (2012)

8. Y. Tamaki, K. Watanabe, K. Koike, et al. Faraday Discuss. 155, 115 (2012)

9. H. Tsubaki, A. Sekine, Y. Ohashi, et al. J. Am Chem. Soc. 127, 15544 (2005)

10. G. L. Closs, J. R. Miller, Science 240, 440 (1988)

11. J.J. Regan, S.M. Risser, D.N. Beratan, J.N. Onuchic, J. Phys. Chem. 9713083 (1993)

12. J. N. Onuchic, D. N. Beratan, J. Chem. Phys. 92, 722 (1990) 